\title{
OXPEMUL, CAMPECHE: \\ UN ACERCAMIENTO A LA TEMPORALIDAD DE SU ASENTAMIENTO
}

\author{
María del Rosario Domínguez Carrasco
}

Centro de Investigaciones Históricas y Sociales, Universidad Autónoma de Campeche

Leydi del Carmen Puc Tejero

Facultad de Ciencias Antropológicas, Universidad Autónoma de Yucatán

\author{
William J. Folan Higgins \\ Centro de Investigaciones Históricas y Sociales, \\ Universidad Autónoma de Campeche
}

\begin{abstract}
Resumen: Las recientes excavaciones realizadas en 20 pozos estratigráficos practicados dentro de un área de $9 \mathrm{~km}^{2}$ alrededor de los grupos principales del asentamiento prehispánico de Oxpemul, en la región del Petén, han revelado importante información respecto a la ocupación del mismo, y evidencian una secuencia cronológica que va del periodo Preclásico Medio al Clásico Tardío. El estudio de dichos materiales proporcionará elementos que permitirán su comparación con otros procedentes de sitios de las regiones de Río Bec y del Petén.
\end{abstract}

Palabras Clave: Oxpemul, arqueología, cerámica, cultura maya, arquitectura.

ABSTRACT: Recent excavations in 20 stratigraphic wells within an area of 9 squared kilometers surrounding the main groups in the Prehispanic site of Oxpemul (Petén) have revealed some important data concerning the chronological sequence of its occupation from the Middle Preclassic to the Late Classic. Materials will allow a comparative study with some materials from archaeological sites in Río Bec and Petén.

KEYwords: Oxpemul, Archaeology, ceramics, Maya culture, Architecture.

RECEPCIÓN: 31 de agosto del 2010.

ACEPTACIÓn: 11 de noviembre del 2010. 



\title{
OXPEMUL, CAMPECHE: \\ UN ACERCAMIENTO A LA TEMPORALIDAD \\ DE SU ASENTAMIENTO
}

\author{
María del Rosario Domínguez Carrasco \\ Centro de Investigaciones Históricas y Sociales, \\ Universidad Autónoma de Campeche \\ Leydi del Carmen Puc Tejero \\ Facultad de Ciencias Antropológicas, \\ Universidad Autónoma de Yucatán \\ WiLliam J. Folan Higgins \\ Centro de Investigaciones Históricas y Sociales, \\ Universidad Autónoma de Campeche
}

\section{Introducción}

Oxpemul se localiza aproximadamente a $30 \mathrm{~km}$ al noreste de Calakmul. Su ubicación geográfica lo vuelve un sitio estratégico, por encontrarse cerca de los límites de dos estados regionales del periodo Clásico, el de Río Bec al norte y el de Calakmul al sur (figura 1). Empero, de acuerdo con el modelo de organización territorial de las Tierras Bajas Mayas del sur, establecido por Flannery (1975), Marcus (1973) y Adams (1986), Oxpemul figura como una ciudad tributaria de Calakmul durante el Clásico Tardío.

Durante los meses de octubre y noviembre del 2009, fueron realizadas excavaciones en 20 pozos estratigráficos en los alrededores de los dos grupos principales del asentamiento prehispánico de Oxpemul: el Grupo Norte y el Grupo Suroeste. Dichos pozos fueron distribuidos dentro del área que abarca el plano general del sitio de $9 \mathrm{~km}^{2}$ y estuvieron asociados, en la mayoría de los casos, a grupos arquitectónicos significativos. Una minoría de estos fue situada en asociación con rasgos hidráulicos como canales y represas.

La finalidad de planear la excavación de pozos en dicha área respondió a la inquietud de conocer a través de los materiales cerámicos, además de la temporalidad, el desarrollo cultural de la ocupación de Oxpemul en relación con sus dos grupos principales, así como su vínculo con las regiones aledañas de Río Bec hacia el norte, Calakmul hacia el sur y el Usumacinta al oeste (Domínguez Carrasco, 1994; 2008a). Todos los pozos, de 2 por $2 \mathrm{~m}$, se ubicaron en lugares estratégicos, tales como el centro de patios cerrados, en la parte posterior de 


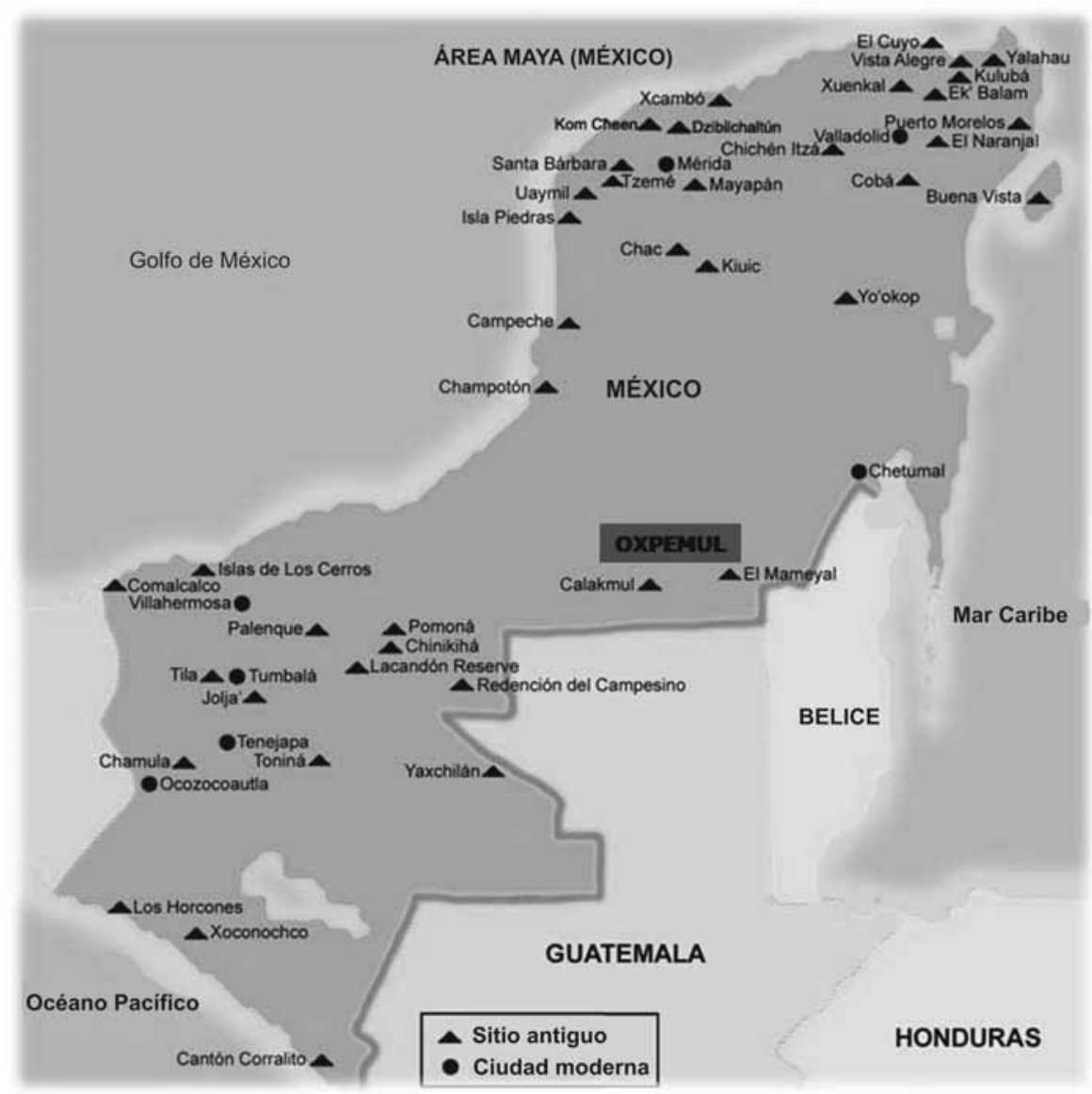

Figura 1. Localización geográfica de Oxpemul

estructuras o fuera de los patios cerrados, siempre con la finalidad de encontrar evidencias que nos pudieran proporcionar mayor información.

Es importante mencionar que muy raras veces se tuvo la posibilidad de registrar una estratigrafía larga. En la mayoría de los casos, debajo de la capa de humus, de 20 a $40 \mathrm{a} \mathrm{cm}$ en promedio, se obtuvo una capa más de tierra de color más claro de $40 \mathrm{~cm}$ en promedio; se llegó finalmente a la roca madre, excepto en algunos casos en donde se alcanzaron gruesas capas estériles. En otros casos, la roca madre apareció por debajo de la capa humítica y sólo en casos excepcionales se registraron vestigios de pisos de estuco. Únicamente en el pozo que fue practicado en el Grupo Norte se registraron elementos arquitectónicos correspondientes a una ocupación anterior, como la presencia de un basamento con esquinas redondeadas correspondiente al Clásico Temprano. 


\section{Antecedentes}

Como sabemos, la existencia de ciudades-estado y estados en Mesoamérica durante el periodo Clásico Temprano respondió a la evolución de complejos sistemas políticos; ello propició la existencia de fronteras culturales y zonas de amortiguamiento entre las diferentes entidades políticas. William Ferguson y Richard Adams (2001) han establecido que el Estado Regional de Río Bec (figura

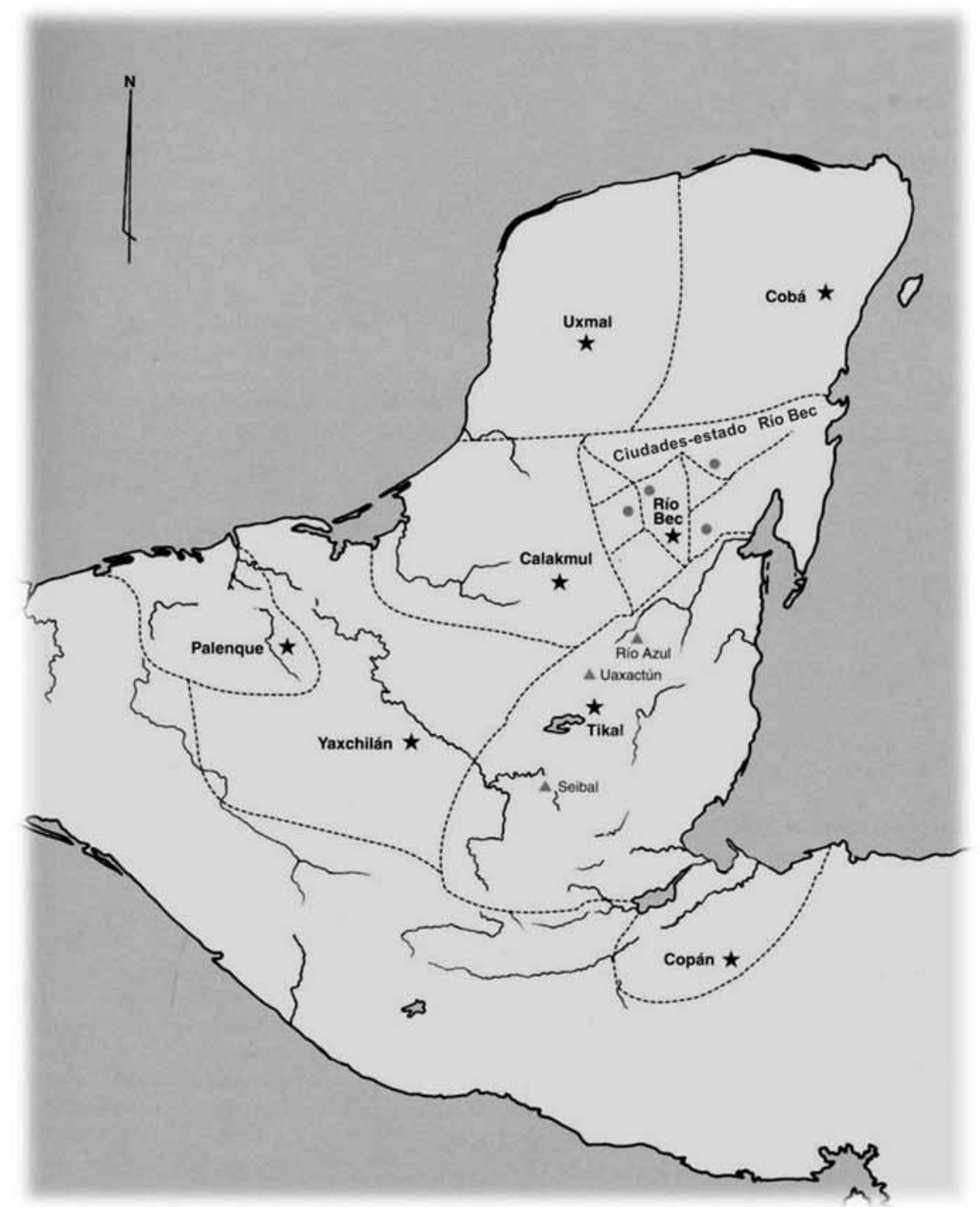

Figura 2. Localización del Estado Regional de Río Bec (Ferguson y Adams, 2001) 
2) durante el periodo Clásico Temprano, a pesar de haberse considerado como una ciudad-estado independiente, debió formar parte de la esfera de Calakmul. En contraste, mostró una marcada autonomía durante el Clásico Tardío, como se observa en Becán, que surge como una grandiosa ciudad fortificada en este periodo.

Hasta el momento, es escasa la información que se tiene respecto a estudios realizados en el área que ocupó el Estado Regional de Río Bec. Se cuenta con un completo estudio sobre la tipología cerámica de Becán y su desarrollo cronológico a través de sus diferentes etapas culturales realizado por Ball (1977), mismo que ha proporcionado una buena fuente para estudios comparativos a nivel regional.

Otro trabajo importante es el de Philippe Nondédéo (1999), quien en el año de 1997 realizó un reconocimiento arqueológico de aproximadamente $400 \mathrm{~km}^{2}$ hacia al este de Becán y norte de Oxpemul, entre los sitios de Balamkú y Manos Rojas, en un área de más o menos $40 \mathrm{~km}$ de largo por $10 \mathrm{~km}$ de ancho. Su finalidad fue identificar lo que pudiera ser un corredor cultural entre la regiones de Río Bec y el Petén, basado en la presencia de pequeños sillares cuadrados, característicos del estilo Río Bec asociados con el periodo Clásico Terminal. Dicho elemento arquitectónico fue registrado en varios de los sitios visitados dentro de un área considerada por Nondédéo como fuera de los límites tradicionales del estilo Río Bec durante este periodo, sugiriendo traslapes, tanto espaciales como cronológicos, entre las influencias Río Bec y Petén. Asimismo, realizó una serie de pozos estratigráficos en 11 sitios de mayor importancia de acuerdo con su arquitectura; su finalidad era contar con una secuencia cronológica de la ocupación de la región bajo estudio.

En el año 2002, y con una perspectiva de trabajo de cinco años, Dominique Michelet y M. Charlotte Arnauld iniciaron una serie de nuevas investigaciones arqueológicas en el sitio de Río Bec con la finalidad de entender el apogeo del estilo homónimo fechado entre el 600 y el 1000 d.C. (Michelet, Arnauld y Nondédéo, 2007; Arnauld, Dzul Góngora y Déodat, 2010). El estudio de los materiales cerámicos determinó una ocupación desde el Preclásico Medio hasta el Posclásico Tardío, cuando ocurrió el abandono casi completo de la zona (Dzul Góngora y Taladoire, 2010; Nondédéo y Dzul Góngora, 2010). Asimismo, los reconocimientos en el sitio aportaron el registro de nuevos grupos arquitectónicos y los estudios epigráficos aportaron la identificación de un glifo emblema, que parece ser el primero identificado en toda la región de Río Bec (Michelet, Arnauld y Nondédéo, 2007).

De manera paralela, en el año 2005 William Folan (Folan et al., 2005; 2008), apoyando la idea de Ferguson y Adams (2001) sobre la existencia de zonas de amortiguamiento entre los estados regionales mesoamericanos, considera al asentamiento prehispánico de Oxpemul como un sitio que delimita una frontera entre dos de los Estados Regionales más importantes del periodo Clásico maya, el de Río Bec al norte y el de Calakmul al sur. Lo anterior se basó en la información proporcionada por el análisis químico y arqueológico de materiales 
cerámicos procedentes de Calakmul y su Estado Regional (Domínguez Carrasco, 2004; 2008b).

En el año 2006 se inició una serie de trabajos de investigación en el sitio arqueológico de Oxpemul encabezada por William Folan; entonces se elaboró un plano de $9 \mathrm{~km}^{2}$ con el registro y levantamiento de 1400 estructuras (Folan et al., 2008) (figura 3). Asimismo, se realizó un estudio epigráfico de los monumentos esculpidos presentes en el sitio, consistentes en 23 estelas (19 localizadas en el Grupo Norte y cuatro localizadas en el Grupo Suroeste) y 21 altares, mismo que permitió identificar el glifo emblema del sitio, además de aportar importante información sobre la presencia de diferentes gobernantes, así como datos relevantes respecto a la temporalidad de dichos monumentos y de los textos e iconografía de los mayas antiguos de Oxpemul (Robichaux y Pruett, 2005; 2008; Robichaux, 2009). De igual manera, se realizaron análisis fisicoquímicos de pigmentos provenientes de las estelas y altares con la finalidad de entender el desarrollo de las tradiciones pictóricas en la región (Domínguez et al., 2009).

A pesar de que hoy en día conocemos algunos datos sobre el aspecto social y político de la nobleza de Oxpemul, carecíamos de información relacionada con la evolución del asentamiento en la periferia de los grupos arquitectónicos mayores, los cuales se localizan estratégicamente sobre dos mesetas. Por ello, el sondeo de los 20 pozos estratigráficos y el material arqueológico recuperado fueron indicadores claves para entender el desarrollo del asentamiento durante los diferentes periodos cronológicos.

\section{La secuencia de Oxpemul}

Los materiales cerámicos analizados reportaron una ocupación desde el Preclásico Medio hasta el Clásico Terminal. La mayor ocupación ocurrió en la periferia de los grupos principales durante el periodo Clásico Temprano y en los mismos grupos durante el Clásico Tardío (figura 4). Un dato que vale la pena resaltar es la presencia de una mayor cantidad de tipos cerámicos correspondientes al periodo Preclásico Tardío en comparación con los tipos procedentes del Clásico Terminal, lo cual descubrió un patrón marcadamente diferente al observado en la región de Calakmul (Domínguez Carrasco, 1994; 1995).

\section{Preclásico Medio}

La ocupación del Preclásico Medio se concentra principalmente hacia el sur, en grupos habitacionales cercanos a la meseta donde descansa el Grupo Suroeste. En el cuadrante B-III se localiza uno de los grupos arquitectónicos más relevantes para este periodo formado por dos conjuntos adyacentes de estructuras abovedadas que se ordenan en patios cerrados y hundidos. La importancia de este conjunto 


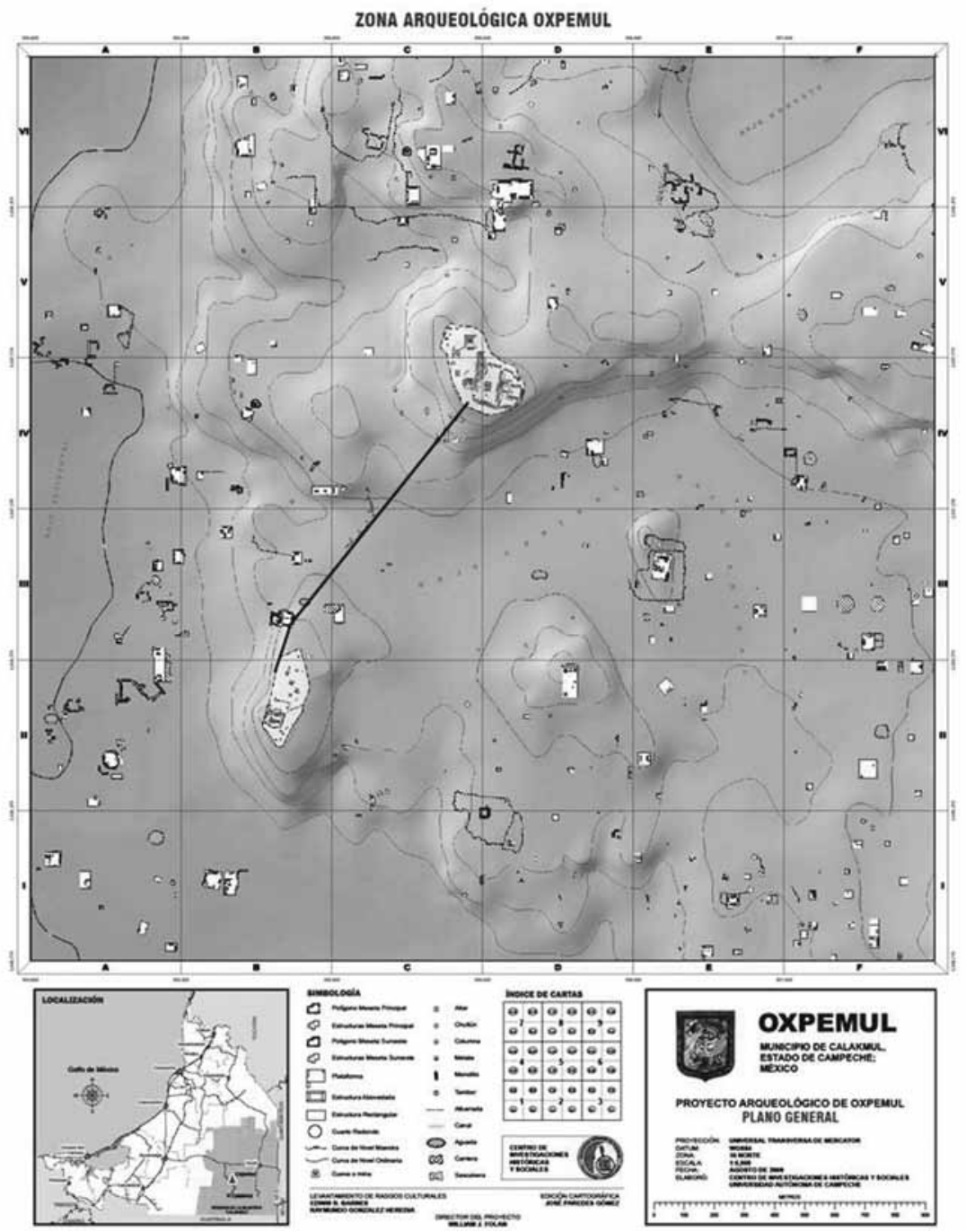

Figura 3. Plano de Oxpemul de $9 \mathrm{~km}^{2}$ (Barnes y González, 2008)

es la conexión que tiene con un sacbé, el cual comunica los dos grupos principales de Oxpemul: el Norte y el Suroeste. Todo lo anterior nos permite inferir que muy probablemente este camino fue construido desde estas fechas tan tempranas.

Es precisamente en el Grupo Suroeste donde los materiales evidenciaron también una definida ocupación durante el Preclásico Medio, por lo que podemos confirmar la probable comunicación entre ambos, así como establecer que este grupo funcionó como el principal centro cívico ceremonial de Oxpemul durante este periodo. 


\section{PORCENTAJE DE CERÁMICA POR PERIODO}

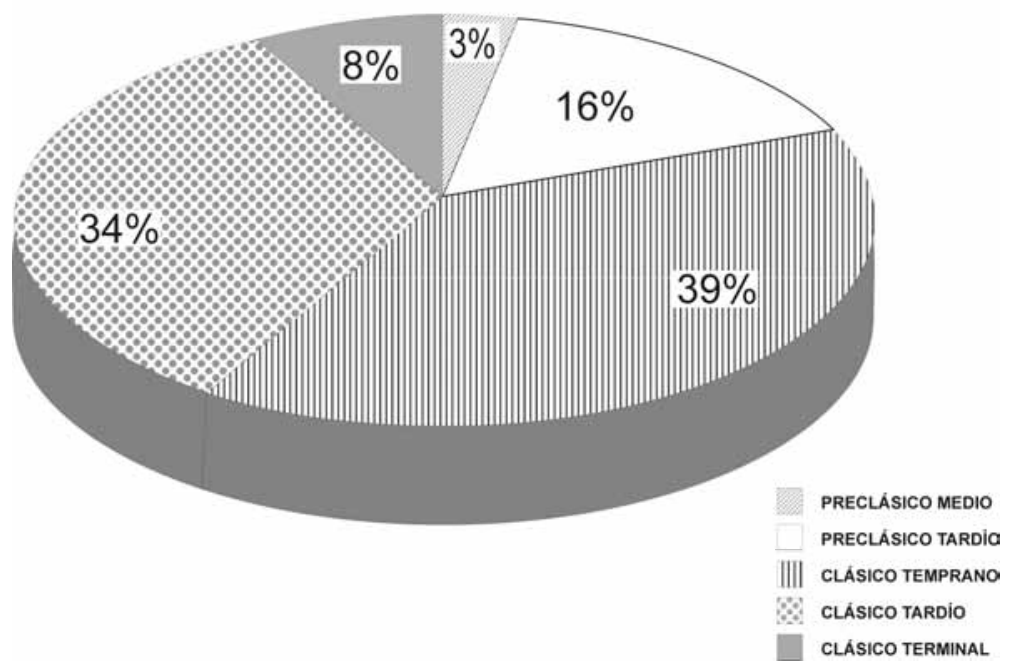

Figura 4. Porcentaje de la cerámica de Oxpemul por periodos cronológicos

En el cuadrante E-III, ubicado hacia el este del plano general, se localiza (de acuerdo con los materiales cerámicos), otro de los rasgos arquitectónicos más representativos de este periodo. Se trata de una gran plataforma de aproximadamente $100 \mathrm{~m}$ de largo por $60 \mathrm{~m}$ de ancho, construida en dos diferentes niveles, sobre la que descansa una estructura mayor de aproximadamente $7 \mathrm{~m}$ de altura, asociada con estructuras menores de forma rectangular. Tal plataforma se encuentra delimitada por una albarrada, cuya entrada está señalada con un monolito.

Existe hacia el norte, y como una de las escasas evidencias de ocupación del Preclásico Medio, un gran complejo arquitectónico en los cuadrantes D-V y D-VI que consiste en una plataforma en forma de $L$ invertida, de aproximadamente $160 \mathrm{~m}$ de largo por $140 \mathrm{~m}$ de ancho; sobre esta descansan estructuras de forma rectangular que delimitan dicha plataforma. Tal vez el elemento cultural que justifica la construcción de dicho conjunto arquitectónico sea la proximidad a dos rasgos culturales de gran relevancia: una cueva/mina formada por 10 cámaras, cuyo sondeo registró la presencia de una extensa ocupación que va desde el Preclásico Medio hasta el Clásico Terminal; y una cueva con una entrada de tiro de aproximadamente $6 \mathrm{~m}$ y cinco cámaras en su interior, algunas con puertas a base de piedras monolíticas, que por sus características pudo haber funcionado como tumba para entierros múltiples o como escondites.

Los materiales cerámicos de este periodo corresponden en su mayoría al grupo cerámico Joventud Rojo. 


\section{Preclásico Tardío}

Se observa una continuidad de los grupos arquitectónicos del Preclásico Medio y la construcción de algunos más a base de grandes plataformas que soportan estructuras con base cuadrangular y rectangular, así como estructuras rectangulares que se ordenan formando pequeñas plazas. A pesar de que las principales evidencias de ocupación continúan en el sur, los materiales cerámicos nos muestran un desplazamiento del asentamiento hacia el septentrión, donde se halla la meseta del Grupo Norte. La finalidad fue construir un segundo centro cívico ceremonial, con grandes templos descansando sobre basamentos con esquinas redondeadas.

Los materiales cerámicos comienzan a exhibir una mayor relación con tipos de la región de Río Bec, como la variedad Peine del grupo Flor Crema, la variedad Añejo del Grupo Sapote y cerámica de grupo Maxcanu. Asimismo, continúa la presencia de materiales representativos de la región del Petén caracterizados por los grupos Sierra, Sapote y Polvero.

Es importante resaltar la abundancia de pastas y de acabado de superficie observado en el tipo cerámico Sierra Rojo, pues generalmente este se ha caracterizado por mostrar una técnica de manufactura uniforme. Esta diversidad en el color de las pastas, en el grosor de las paredes de las vasijas y en el acabado de superficie fue reportada asimismo por Ball (1977) en el sitio de Becán.

\section{Clásico Temprano}

El periodo Clásico Temprano muestra el momento de máxima extensión del asentamiento, lo cual se refleja en la presencia de una mayor variedad de tipos cerámicos. De acuerdo con los estudios epigráficos realizado por Robichaux y Pruett (2008), es en el Grupo Suroeste en donde se encuentran las estelas más tempranas del sitio (Estela 22 y Estela 23), posiblemente del siglo IV o inicios del siglo v. Ello nos indica que para este momento tanto este grupo, tal vez con algunas remodelaciones en los edificios monumentales, como el Grupo Norte se desempeñaban como dos espacios de gran importancia, otorgándole a Oxpemul cierto grado de autonomía con respecto a las entidades políticas de Río Bec y Calakmul para manifestar su poder social y político.

De acuerdo con la cerámica, existe un equilibrio entre la presencia de tradiciones de la región de Río Bec y del Petén que nos confirma lo que Richard Adams (1986) mencionó respecto a la influencia cultural de la esfera de Calakmul en el Estado Regional de Río Bec (caracterizado como un periodo de máxima autonomía de este último).

Existe la presencia de una gran variedad de pastas en la manufactura de vasijas, lo cual nos sugiere una mayor explotación de fuentes de arcilla en lugares próximos a Oxpemul. Se observa asimismo una mayor diversidad en la producción de vasijas utilitarias sin engobe, específicamente en las de tipo estriado. 
En lo que respecta a las utilitarias con engobe, sobresale la fabricación en gran escala de vasijas de la variedad Flamboyán del grupo cerámico Águila Naranja, las cuales superan las frecuencias reportadas por Ball (1977) para Becán. No obstante, comparte su presencia en cantidades similares con la variedad Águila de la región del Petén. Por la ausencia de esta vajilla en la región de Calakmul, podemos afirmar su restringida manufactura y distribución para la región de Río Bec.

\section{Clásico Tardío}

Durante el periodo Clásico Tardío, al igual que la mayoría de los sitios de las Tierras Bajas Mayas, Oxpemul destaca por una ocupación importante. En este momento, y por la arquitectura observable en el Grupo Norte, parece que esta meseta se convierte en el principal espacio cívico-ceremonial del asentamiento, al que se llegaba por medio de un sacbé, de aproximadamente $1300 \mathrm{~m}$ de largo, que se comunicaba con el Grupo Suroeste. Esta meseta fue aprovechada en su totalidad con la construcción y remodelación de 56 estructuras agrupadas en plazas, incluyendo un juego de pelota, un grupo E, además de 21 estelas y 20 altares fechados entre el 731 d.C. y el 830 d.C.

La cerámica descubre que en esta época continúa la expansión del asentamiento hacia el norte, sin dejar la ocupación de los grupos habitacionales localizados en el sur; asimismo, se identifica la construcción de nuevos grupos arquitectónicos que buscan la cercanía a fuentes de agua, como aguadas y canales.

El sistema constructivo continúa el mismo patrón, a base de edificios cuadrangulares y rectangulares sobre grandes plataformas, así como estructuras rectangulares ordenadas en patios cerrados y en ocasiones delimitados por extensas albarradas.

Los mayores porcentajes de cerámica están representados por los grupos Tinaja, Becanchén, Cambio y Encanto, y con menor frecuencia por el Infierno. De ellos cuatro corresponden a esferas cerámicas del Petén y uno muestra influencia de la región de Río Bec.

\section{Clásico Terminal}

En este periodo ocurrió una disminución de la ocupación en el asentamiento, relacionada tal vez con movimientos de la población hacia otras regiones en busca de mejores fuentes de subsistencia. La mayor concentración de materiales se localizó cerca de $1 \mathrm{~km}$ hacia el norte del Grupo Norte (en el mismo conjunto que presentó una ocupación ininterrumpida desde el Preclásico Medio) y hacia el sureste del mismo grupo, en un complejo arquitectónico que se localiza a una distancia de $700 \mathrm{~m}$ en línea recta y que de igual manera reportó una ocupación desde el Preclásico Medio. Cerca de este último fue registrado recientemente un 
sacbé inacabado, que bien pudiera estar relacionado con el desplazamiento de los oxpemuleños hacia el sur, inclusive hacia Calakmul; al contrario de lo que se ha observado en Oxpemul y otros sitios de la región de Río Bec, los materiales cerámicos (Domínguez Carrasco, 2004) revelan una fuerte ocupación de Calakmul durante este periodo. También se llevaron al cabo entonces grandes remodelaciones arquitectónicas en la fachada principal de la Estructura II, considerada como uno de los más importantes edificios cívico-ceremoniales durante los periodos Clásico Temprano y Clásico Tardío (Folan et al., 2007; Gunn et al., 2009).

Los materiales culturales, en asociación con las remodelaciones arquitectónicas, nos permiten inferir la práctica de diversas actividades relacionadas con la vida cotidiana. Todo esto hace posible percibir un cambio total en la función del edificio y, por consiguiente, la clara decadencia política y social de Calakmul como la capital de un estado regional.

En cuanto a los materiales cerámicos, estos muestran asimismo una mayor influencia de la región del Petén; predominan los grupos peteneros Tinaja y Máquina, lo cual nos sugirió una mayor filiación de dichas piezas con Calakmul que con Río Bec.

\section{Consideraciones finales}

La situación de Oxpemul parece ajustarse al Modelo Dinámico propuesto por Marcus $(1993 ; 1998)$, quien demuestra la existencia de un sistema dinámico durante el desarrollo de sociedades complejas, con la presencia de cambios en las jerarquías de algunos asentamientos en un momento determinado del proceso cultural (figura 5).

En el Modelo Dinámico, la cúspide de cada ciclo representa un estado maya territorialmente extenso con una jerarquía de asentamientos de por lo menos cuatro niveles, en el cual los tres niveles superiores eran gobernados por señores hereditarios que detentaban el control administrativo, como fue el caso de Calakmul. El punto más bajo de cada ciclo representa la división del gran estado en provincias más pequeñas, semiautónomas o en alianzas indefinidas que en ocasiones presentaban jerarquías de asentamientos de sólo tres niveles, como pudo ser también el caso de Calakmul durante el Clásico Terminal.

En este periodo del Clásico Temprano, Calakmul mostró una presencia regional excepcionalmente fuerte que continuó hasta los inicios del Clásico Tardío, presencia que estuvo también marcada por condiciones climáticas favorables (Gunn, Folan y Robichaux, 1994; 1995); esta expansión se puede constatar por la frecuente y amplia distribución de su glifo emblema en lugares tan lejanos como Piedras Negras en Guatemala, Caracol en Belice y Copán en Honduras (Marcus, 1973; 1976; 1993). En uno de sus periodos de mayor expansión, Calakmul incorporó varias provincias a su gobierno durante los siglos v y vi d.C. (entre las que se encuentra Oxpemul) y formó un gran número de alianzas que la convirtieron 


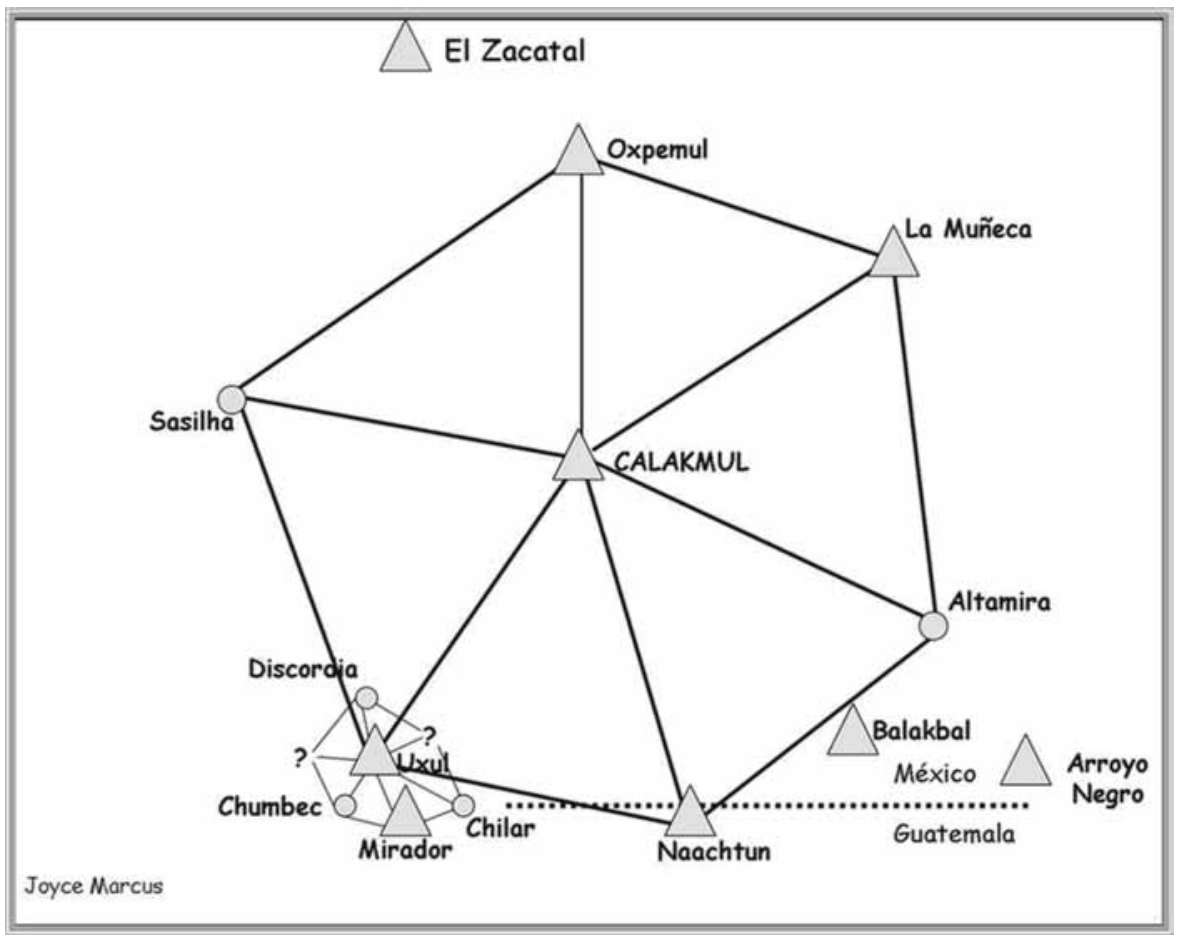

Figura 5. Estado Regional de Calakmul (Marcus, 1973)

en la capital maya más frecuentemente mencionada en monumentos fuera de su región a través de la inscripción de su glifo emblema (Marcus, 1993).

Sin duda alguna, es en este periodo del Clásico Temprano que Oxpemul también llega a su máxima expansión. La presencia del glifo emblema de Calakmul en una de sus estelas más tempranas, fechada en el siglo v (Robichaux y Pruett, 2008; Robichaux, 2009), nos corrobora su estatus como ciudad tributaria administrada bajo el control de Calakmul, la cual debió haber participado en extensas redes y alianzas que sirvieron para fines militares, sociales, políticos, económicos y rituales entre ambas entidades.

Es durante el Clásico Tardío que Oxpemul adquiere una mayor autonomía con respecto a ambas capitales regionales, la de Río Bec y la de Calakmul. Las fechas identificadas respecto al reinado de los gobernantes de Oxpemul (731 d.C.) coinciden con el final de la dinastía Kan. La Estela 9 de Oxpemul (figura 6) indica la fuerte posibilidad de que esta última ciudad halla ganado alguna batalla contra Calakmul alrededor del 751 d.C., además de ser el momento en que la misma urbe fue derrotada por Tikal y y su poder comenzaba a disminuir.

De igual manera, el análisis químico realizado con anterioridad a pigmentos procedentes de Oxpemul y Calakmul reveló patrones diversos respecto a la pre- 

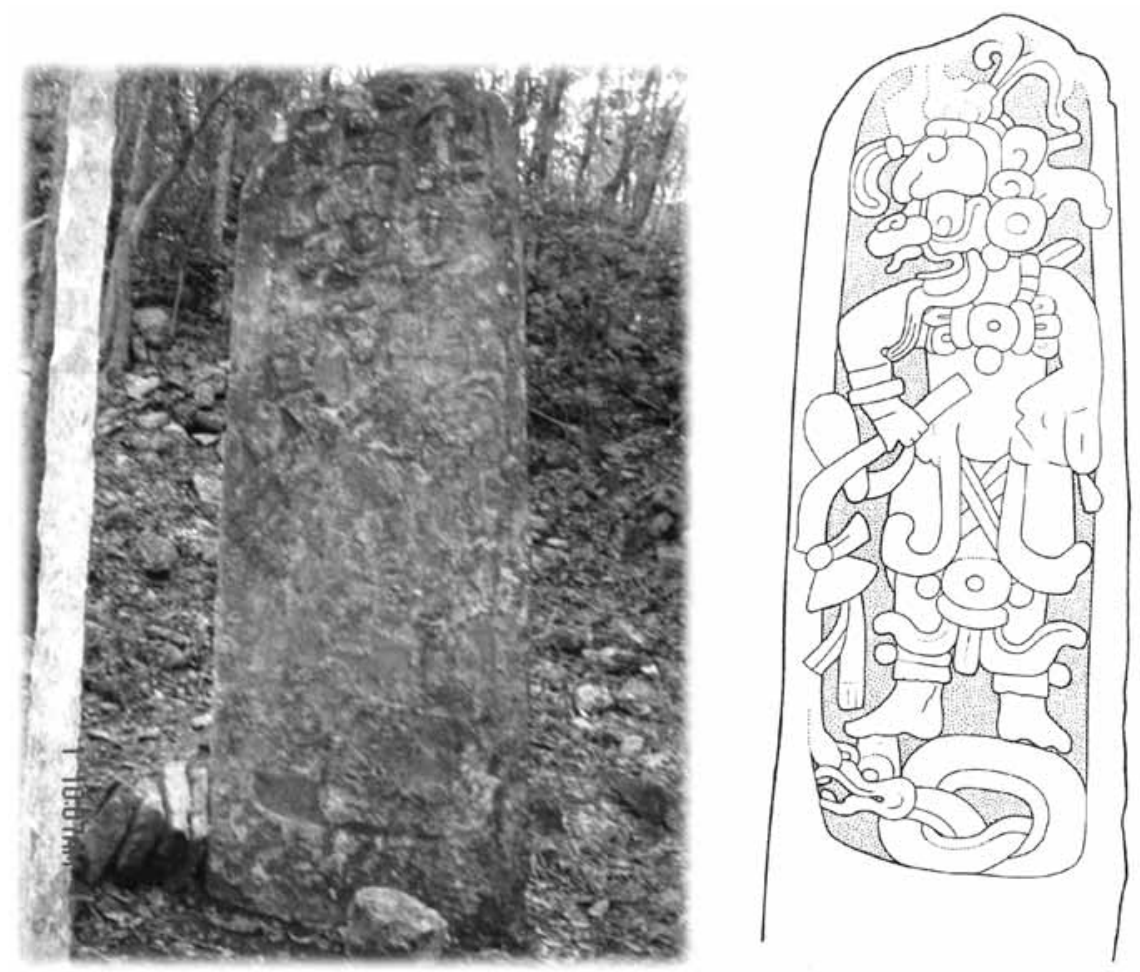

Figura 6. Estela 9 de Oxpemul, que indica la posibilidad de que este sitio halla ganado alguna batalla contra Calakmul alrededor del 751 d.C. (Robichaux y Pruett, 2008; Robichaux, 2009).

Dibujo de Nikolai Grube

sencia de ciertos componentes químicos que formaron parte de las recetas de los pigmentos aplicados como recubrimiento a las estelas y altares de ambos sitios (Domínguez et al., 2009; Espinosa et al., 2008). Este hecho coincide con los resultados obtenidos a través del presente estudio.

Tenemos que la arcilla paligorskita, que formó parte de la preparación de la receta para la obtención del pigmento rojo, sólo se encontró presente en el colorante procedente de las estelas de Oxpemul fechadas en el periodo Clásico Tardío. En cambio, estuvo ausente en los pigmentos analizados de Calakmul correspondientes al mismo periodo. Lo anterior nos indica, al igual que los materiales cerámicos, la diferencia de patrones culturales entre ambas ciudades; esto nos permite percibir durante este periodo la escasa relación de Oxpemul como ciudad dependiente de Calakmul.

Retomando lo anterior y continuando con el Modelo Dinámico, Calakmul muestra una curva descendente como capital regional debido a un ciclo de disolución por la aparente pérdida de Oxpemul como ciudad tributaria. En tanto, 
Oxpemul adquiere independencia y una mayor autonomía manifiestas en la identificación del glifo emblema hasta el siglo ıx (figura 7), momento en que disminuyen las actividades de su población y se traslada hacia el sur.

Futuras excavaciones extensivas en el Grupo Norte y en el Grupo Suroeste nos deberán proporcionar una mayor información respecto a este momento de gran relevancia social y política que Oxpemul experimentó a través del periodo Clásico Tardío. Por el momento, los sondeos realizados en la periferia nos ha ofrecido un acercamiento a la temporalidad y desarrollo del asentamiento dentro de un área de $9 \mathrm{~km}^{2}$, en el que se registra la mayor expansión del mismo durante el periodo Clásico Temprano.
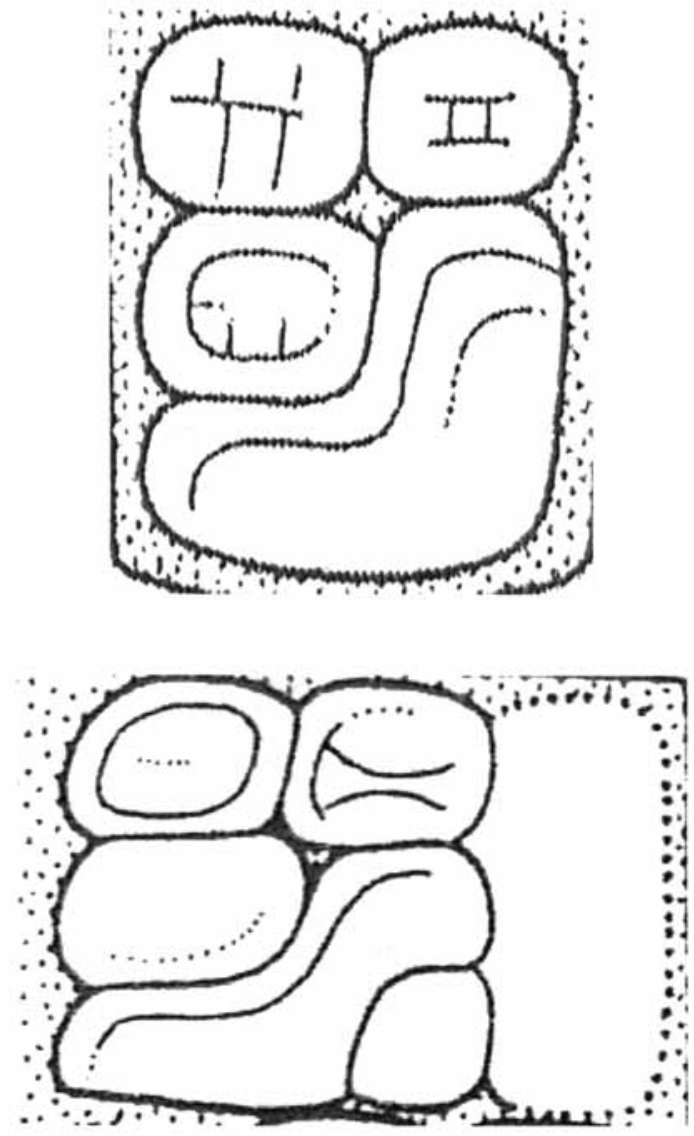

Figura 7. Glifos emblema de Oxpemul (Robichaux y Pruett, 2008) 


\section{BIBLIOGRAFÍA}

Adams, Richard E. W.

1986 "The Ceramics of Rio Azul, 1984”, Rio Azul Reports. Number 2. The 1984 Season, pp. 46-53. San Antonio, Texas: Center of Archaeological Research, University of Texas.

Arnauld, Charlotte, Sara Dzul Góngora y Laure Déodat

2010 "Evolución de la ocupación en el Grupo B de Río Bec", La Península de Yucatán: investigaciones recientes y cronologías alternativas, pp. 135-154. Campeche: Universidad Autónoma de Campeche.

Ball, Joseph W.

1977 The Archaeological Ceramics of Becán, Campeche, México. Nueva Orleans: Middle American Research Institute/Tulane University (Publication 43).

Barnes, Edwin, Raymundo González Heredia y William J. Folan

2008 "Las ruinas de Oxpemul, Campeche: su corte real y su huella urbana dentro de la Cuenca de Calakmul. El mapa y su base de datos". Informe de actividades del proyecto Oxpemul, Campeche: Frontera Cultural Prehispánica de las Tierras Bajas Mayas del Periodo Clásico. Manuscrito. Universidad Autónoma de Campeche, Centro de Investigaciones Históricas y Sociales.

Domínguez Carrasco, María del Rosario

1994 Calakmul, Campeche. Un análisis de la cerámica. Campeche: Universidad Autónoma de Campeche (Colección Arqueología, 4).

1995 "La cerámica de Calakmul, Campeche: una visión de su secuencia cronológica y cultural”, Los Investigadores de la Cultura Maya, 3 (II): 503-521. Campeche: Universidad Autónoma de Campeche.

2004 "Fuentes, producción y consumo de cerámica en el Estado Regional de Calakmul, Campeche. Un enfoque sociopolítico". Tesis de doctorado en Estudios Mesoamericanos, México: Universidad Nacional Autónoma de México, Facultad de Filosofía y Letras.

2008a Análisis químico y sociopolítico de producción cerámica preshispánica en la región de Calakmul, Campeche. Campeche: Universidad Autónoma de Campeche (Colección Arqueología, 6).

2008b "Informe del análisis de los materiales cerámicos del proyecto arqueológico: Oxpemul, Campeche: Frontera cultural prehispánica de las Tierras Bajas Mayas del periodo Clásico”. Informe Técnico. Campeche: Universidad Autónoma de Campeche, Centro de Investigaciones Históricas y Sociales.

Domínguez Carrasco, María del Rosario y Manuel E. Espinosa Pesqueira

2008 "Preliminary Analysis of Maya Prehispanic Pigments. A Chemical Characterization Study", ponencia presentada en la 73rd Annual Meeting of the Society for American Archaeology, 26-30 de marzo del 2008. Vancouver, Canada.

Domínguez Carrasco, María del Rosario, Manuel E. Espinosa Pesqueira y William J. Folan Higgins

2009 "Los materiales arqueológicos de Oxpemul, Campeche y su contexto re- 
gional”, Los Investigadores de la Cultura Maya, 18 (I): 253-268. Campeche: Universidad Autónoma de Campeche.

Dzul Góngora, Sara y Eric Taladoire

2010 "Espacio y cronología en la periferia de Río Bec", La Península de Yucatán: investigaciones recientes y cronologías alternativas, pp. 121-134. Campeche, México: Universidad Autónoma de Campeche.

Espinosa Pesqueira, Manuel E., María del Rosario Domínguez Carrasco, Sandra Zetina, María Eufemia Fernández, José Luis Ruvalcaba Sil y Jesús Arenas Alatorre,

2008 "Physical and Chemical Analysis of Mayan Prehispanic Paintings", ponencia presentada en el XVII International Materials Research Congress, 18-21 de agosto de 2008. Cancún, México.

Ferguson, William M., Richard E. W. Adams

2001 Mesoamerica's Ancient Cities. Aerial Views of Pre-Columbian Ruins in Mexico, Guatemala, Belize, and Honduras. Alburquerque, Nuevo México: University of New Mexico Press.

Flannery, Kent V.

1975 La evolución cultural de las civilizaciones. Barcelona: Anagrama.

Folan Higgins, William, Ivan Šprajc, Raymundo González Heredia, Hubert R. Robichaux, María del Rosario Domínguez Carrasco, Abel Morales López, Candace Pruett y Joel D. Gunn

2005 "Las ruinas de Oxpemul, Campeche, México: una corte real fortificada en la frontera norte entre el Estado Regional de Calakmul y el Rio Bec", Los Investigadores de la Cultura Maya, 13 (II): 475-486. Campeche: Universidad Autónoma de Campeche.

Folan Higgins, William, Abel Morales López, Raymundo González Heredia, María del Rosario Domínguez Carrasco, Armando Anaya Hernández y Joel D. Gunn

2007 "Calakmul, Campeche: el desarrollo de la estructura II desde el Clásico Temprano hasta sus últimos días durante el Clásico Terminal”, Los Investigadores de la Cultura Maya, 15 (I): 137-145. Campeche: Universidad Autónoma de Campeche.

Folan Higgins, William J., Raymundo González Heredia, Hubert Robichaux, Edwin Barnes, Abel Morales López, Armando Anaya Hernández, Pedro Zamora Crescencio, María del Rosario Domínguez Carrasco, Joel D. Gunn y Ciriaco Requena S.

2008 "Las ruinas de Oxpemul, Campeche, México: su mapa y avances en el estudio de su patrón de asentamiento, textos jeroglíficos, cerámica y vegetación”, Los Investigadores de la Cultura Maya, 16 (I): 107-134. Campeche: Universidad Autónoma de Campeche.

Gunn, Joel D., William J. Folan y Hubert R. Robichaux

1994 "Un análisis informativo sobre la descarga del río Candelaria en Campeche, México: reflexiones acerca de los paleoclimas que afectaron a los antiguos mayas en los sitios de Calakmul y El Mirador", Campeche maya colonial, pp. 
174-197, W. J. Folan (coord.). Campeche: Universidad Autónoma de Campeche, Centro de Investigaciones Históricas y Sociales (Colección Arqueología, 2).

1995 "A Landscape Analysis of the Candelaria Watershed in Mexico: Insights into Paleoclimates Affecting Upland Horticulture in the Southern Yucatan Peninsula Semi-Karst”, Geoarchaeology: An International Journal, 10 (1): 3-42. Estados Unidos: Archaeological Geology Division.

Gunn, Joel D., William J. Folan, María del Rosario Domínguez Carrasco y W. Frank Miller

2009 "Explicando la sustentabilidad de Calakmul, Campeche: eslabones interiores en el sistema de energía del Estado Regional de Calakmul”, Los Investigadores de la Cultura Maya, 18 (I): 13-40. Campeche: Universidad Autónoma de Campeche.

Marcus, Joyce

1973 "Territorial Organization of the Lowland Classic Maya”, Science, 180: 911916. Nueva York: American Association for the Advancement of Science.

1976 Emblem and State in the Classic Maya Lowlands: An Epigraphic Approach to Territorial Organization. Washington, D. C.: Dumbarton Oaks/Harvard University.

1993 "Ancient Maya Political Organization", Lowland Maya Civilization in the Eighth Century A.D., Lowland Maya Civilization in the Eight Century A.D., pp. 111-183. J. A. Sabloff y J. S. Henderson (eds.). Washington, D. C.: Dumbarton Oaks.

1998 "The Peaks and Valleys of Ancient States: An Extension of the Dynamic Model", Archaic States, pp. 59-94, G. M. Feinman y J. Marcus (eds.). Santa Fe, Nuevo México: School of American Research Press.

Michelet, Dominique, M. Charlotte Arnauld y Philippe Nondédéo

2007 "Río Bec, Campeche, al cabo de tres temporadas de investigaciones en el campo (2002-2004): datos preliminares", El patrimonio arqueológico maya en Campeche, pp. 31-158. México: Universidad Nacional Autónoma de México, Instituto de Investigaciones Filológicas, Centro de Estudios Mayas (Cuadernos del Centro de Estudios Mayas, 35).

Nondédéo, Philippe

1999 "Reconocimiento arqueológico en el sureste de Campeche: Resultados preliminaries de las temporadas 1997-1998", Los Investigadores de la Cultura Maya, VII (1): 85-111. Campeche: Universidad Autónoma de Campeche.

Nondédéo, Philippe y Sara Dzul Góngora

2010 "Arquitectura y cronología: hacia un esquema preliminar de evolución en la ocupación de la microrregión de Río Bec”, La Península de Yucatán: investigaciones recientes y cronologías alternativas, pp. 91-120. Campeche: Universidad Autónoma de Campeche.

Robichaux, Hubert R.

2009 "Textos e iconografía de los mayas antiguos en Oxpemul, Campeche: un resumen actualizado", Los Investigadores de la Cultura Maya, 17 (II): 81-92. Campeche: Universidad Autónoma de Campeche. 
Robichaux, Hubert R. y Candace Pruett

2005 "Las inscripciones de Oxpemul, Campeche", Los Investigadores de la Cultura Maya, 13 (I): 29-43. Campeche: Universidad Autónoma de Campeche.

2008 "Investigaciones epigráficas en Oxpemul, Campeche: descubrimientos recientes”, Los Investigadores de la Cultura Maya, 16 (I): 89-105. Campeche: Universidad Autónoma de Campeche. 
